\title{
ELEVAÇÃO DE HORMÔNIO TIREOESTIMULANTE (TSH) APÓS AS LOBECTOMIAS: INCIDÊNCIA E FATORES ASSOCIADOS
}

\author{
THYROID-STIMULATING HORMONE (TSH) RISING FOLLOWING \\ HEMITHYROIDECTOMY: INCIDENCE AND ADJUVANT FACTORS
}

\author{
Vergillius José Furtado de Araújo Filho, TCBC-SP'; Lenine Garcia Brandão, TCBC-SP'ㅎㅁ Dorival Carlucci Jr²; \\ Raquel Ajub Moysés ${ }^{3}$; Marília D'Elboux Guimarães Brescia ${ }^{3}$; Alberto Rosseti Ferraz, TCBC-SP
}

\begin{abstract}
RESUMO: Objetivo: Determinar a freqüência de elevação da dosagem sérica do hormônio tireoestimulante (TSH) em pacientes submetidos à lobectomia da tireóide, em um período de até 12 semanas após a operação, buscando fatores associados à sua ocorrência. Método: Foram analisados retrospectivamente 88 pacientes submetidos à lobectomia da tireóide no Serviço de Cirurgia de Cabeça e Pescoço do Hospital das Clínicas da FMUSP, no período de setembro de 2002 a setembro de 2004. Realizaram-se dosagens de hormônios tireoideanos a partir de quatro semanas após a cirurgia. Excluíram-se os pacientes com dosagens hormonais pré-operatórias alteradas, os casos que necessitaram de totalização da tireoidectomia e também aqueles em que houve perda do seguimento pós-operatório. Foram analisados os dados quanto à idade e ao sexo dos pacientes, quanto à presença de tireoidite no estudo histopatológico da tireóide e quanto ao tempo de aparecimento do hipotireoidismo. A análise estatística dos dados obtidos foi realizada através do teste qui-quadrado de Pearson. Resultados: Dos 88 pacientes, 71 (80,7\%) eram mulheres. A idade média foi de 41,7 anos. Observou-se elevação do TSH em 20 (22,73\%) dos 88 pacientes estudados. Não foi observada diferença estatisticamente significante na incidência de elevação do TSH, quando analisados quanto ao sexo, à idade ou à presença de tireoidite. Conclusão: A elevação do TSH é freqüente após lobectomias da tireóide e ocorre, muitas vezes, precocemente após a cirurgia. Não se encontraram, neste estudo, fatores que pudessem predizer sua ocorrência a curto prazo (Rev. Col. Bras. Cir. 2007; 34(2): 84-87).
\end{abstract}

Descritores: Tireotropina; Hipotireoidismo; Tireoidectomia; Tireoidite.

\section{INTRODUÇÃO}

A ressecção de um dos lobos da glândula tireóide é um procedimento comum na prática clínica do Cirurgião de Cabeça e Pescoço e, ao contrário das tireoidectomias totais e subtotais, o hipotireoidismo após as lobectomias, principalmente no período pós-operatório recente, é pouco freqüente. São poucos os estudos na literatura que determinam a ocorrência dessa disfunção, bem como o manejo clínico desses pacientes. A fim de verificar a ocorrência de elevação sérica do hormônio tireoestimulante (TSH) em um período de até 12 semanas após as lobectomias, e também os fatores relacionados à sua ocorrência, realizamos o presente estudo.

\section{MÉTODO}

Revisaram-se, retrospectivamente, 88 casos de pacientes submetidos à lobectomia da glândula tireóide entre setembro de 2002 e setembro de 2004 na Disciplina de Cirurgia de Cabeça e Pescoço do Hospital das Clínicas da Faculdade de Medicina da Universidade de São Paulo. Adota-se, em nosso Serviço, a técnica de inclusão do istmo da tireóide na lobectomia.. Todos os pacientes estavam com função tireoideana normal no momento da operação e tinham dosagens hormonais realizadas de rotina até 12 semanas após a lobectomia. Em casos de sintomatologia precoce de hipotireoidismo, as dosagens foram realizada mais precocemente, a partir da $4^{\mathrm{a}}$ semana. Foram previamente excluídos os pacientes sem seguimento pós-operatório, com dosagens hormonais pré-operatórias alteradas ou em que foi necessária totalização de tireoidectomia por achado de neoplasia maligna no exame histopatológico. Os pacientes foram analisados quanto ao sexo, à idade e ao achado histopatológico de tireoidite. A análise estatística dos dados obtidos foi realizada através do teste qui-quadrado de Pearson, sendo adotado, como significância estatística, o valor de $p<0,05$.

\footnotetext{
1. Professor Livre-Docente da Disciplina de Cirurgia de Cabeça e Pescoço do Hospital das Clínicas da Faculdade de Medicina da Universidade de São Paulo (DCCP-HCFMUSP)

2. Médico Pós-graduando da Disciplina de Cirurgia de Cabeça e Pescoço do Hospital das Clínicas da Faculdade de Medicina da Universidade de São Paulo (DCCP-HCFMUSP)

3. Médica Residente da Disciplina de Cirurgia de Cabeça e Pescoço do Hospital das Clínicas da Faculdade de Medicina da Universidade de São Paulo (DCCP-HCFMUSP)

4. Professor Titular da Disciplina de Cirurgia de Cabeça e Pescoço do Hospital das Clínicas da Faculdade de Medicina da Universidade de São Paulo (DCCP-HCFMUSP)
}

Recebido em 08/09/2006

Aceito para publicação em 20/11/2006

Conflito de interesses: nenhum

Fonte de financiamento: nenhuma

Trabalho realizado na Disciplina de Cirurgia de Cabeça e Pescoço do Hospital das Clínicas da Faculdade de Medicina da Universidade de São Paulo. 


\section{RESULTADOS}

Desses pacientes, $71(80,68 \%)$ eram mulheres e 17 $(19,32 \%)$ eram homens, em idades que variaram de 8 a 78 anos (média de 41,74 anos)

Dos 88 casos de pacientes submetidos à lobectomia, $20(22,73 \%)$ apresentaram elevação do TSH no período até 12 semanas após a operação (Figura 1). Não houve diferença estatística em relação à distribuição por sexo, idade e presença de tireoidite no exame histopatológico (Tabela 1).

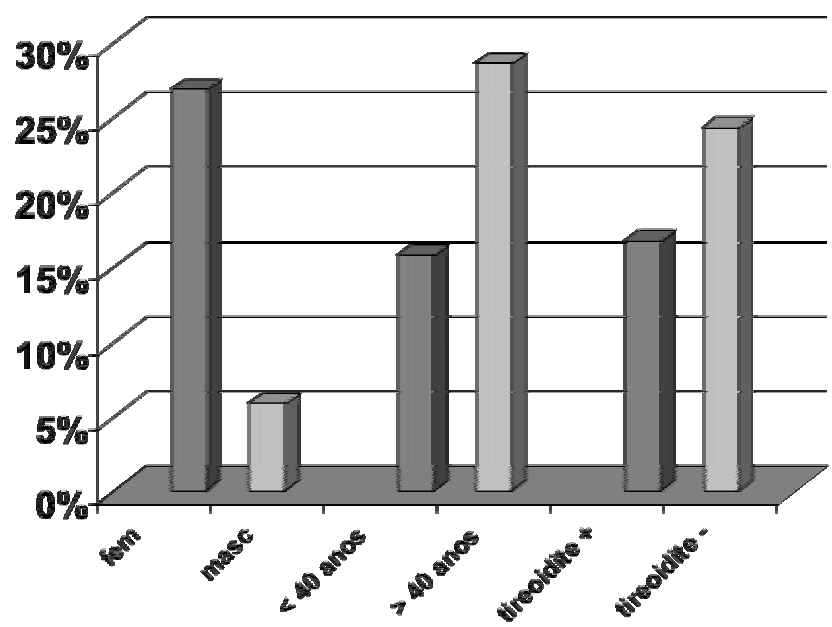

Figura 1 - Ocorrência de hipotireoidismo conforme sexo, idade e presença de tireoidite.

Tabela 1 - Resultados.

\begin{tabular}{llccc}
\hline & & \multicolumn{3}{c}{ Número de pacientes } \\
\cline { 3 - 5 } & & TSH alt & TSH nl & $\boldsymbol{p}$ \\
\hline Sexo & Fem & 19 & 52 & $\mathbf{0 , 1}$ \\
& Masc & 1 & 16 & \\
Idade & $<40$ & 6 & 32 & $\mathbf{0 , 2}$ \\
\multirow{4}{*}{ Tireoidite } & Sim & 3 & 35 & \\
& Não & 17 & 15 & $\mathbf{0 , 7 5}$ \\
& & 30 & 53 & \\
\hline
\end{tabular}

TSH: hormônio tireoestimulante; alt: alterado; nl: normal.

\section{DISCUSSÃO}

As publicações sobre hipotireoidismo como conseqüência de lobectomias são, além de escassas, pouco comparáveis entre si, pelos diferentes perfis de pacientes analisados (Tabela 2).

Sabe-se que o TSH sérico altera-se imediatamente mediante discretas alterações na concentração de hormônios tireoideanos, o que faz de sua dosagem um teste de screening muito sensível para estimativa da função da tireóide ${ }^{1} \mathrm{e}$, nos casos de níveis elevados, um indicativo fiel de hipotireoidismo - clínico ou subclínico - após lobectomias ${ }^{2,3}$. Porém a meiavida da tiroxina é de cerca de sete dias, o que implica em um período aproximado de cinco meias-vidas (cinco semanas) para aferição do TSH a fim de se estimar a função do lobo remanescente $^{4}$. Sabe-se que, em pacientes recebendo tratamento supressor com tiroxina, os níveis séricos de TSH elevam-se e estabilizam-se em cerca de duas a três semanas (no máximo seis semanas) após a interrupção do tratamento ${ }^{5}$.

McHenry $^{4}$ dosou o TSH de 71 pacientes lobectomizados e constatou hipotireoidismo - conceituado como aumento de TSH pelo menos cinco semanas após a operação - em 35\% deles, sendo em 12,3\% com repercussão clínica. Buchanan ${ }^{6}$, também, através de dosagens de TSH, encontrou hipotireoidismo em $24 \%$ dos 158 pacientes submetidos à lobectomia com seguimento mínimo de dois anos. Hedman $^{2}$, estudando 95 pacientes, por um período médio de 15 anos, encontrou 5\% de lobectomizados com hipotireoidismo, caracterizado por alterações hormonais e clínicas. Cheung ${ }^{7}$ avaliou 103 pacientes submetidos à lobectomia e constatou alterações significativas nas dosagens de tiroxina sérica e TSH em três anos após a operação. Estes dois hormônios se alteraram em 13 desses pacientes. Não há diferença na evolução hormonal entre homens e mulheres após as lobectomias ${ }^{4,8,9}$, tal fato foi verificado na nossa série, no entanto, há que se considerar que os pacientes do sexo masculino são em número reduzido, fato que impede conclusões precisas a esse respeito.

Para este estudo, analisaram-se as dosagens de TSH em curto período após a tireoidectomia. Sua elevação não foi denominada como hipotireoidismo, já que há controvérsia em se classificar assim quadros de elevação de TSH precocemente após cirurgia.

Tabela 2 - Ocorrência de hipotireoidismo em diferentes séries de literatura.

\begin{tabular}{|c|c|c|c|c|}
\hline Autor & Ano & $\begin{array}{c}\text { Definição de } \\
\text { hipotireoidismo }\end{array}$ & $\begin{array}{c}\text { Ocorrência de } \\
\text { hipotireoidismo }\end{array}$ & $\begin{array}{c}\text { Período da análise } \\
\text { pós lobectomia }\end{array}$ \\
\hline Cheung $^{7}$ & 1986 & Alteração hormonal & $12,6 \%$ & 3 anos \\
\hline Hedman $^{2}$ & 1986 & Alterações hormonal e/ou clínica & $5 \%$ & 15 anos \\
\hline McHenry $^{4}$ & 2000 & Alteração hormonal & $35 \%$ & $=5$ semanas \\
\hline Buchanan $^{6}$ & 2001 & Alteração hormonal & $24 \%$ & $=2$ anos \\
\hline Piper $^{13}$ & 2005 & Alteração hormonal & $18 \%$ & $>37$ meses \\
\hline Miller $^{9}$ & 2006 & Alteração hormonal & $27 \%$ & $>8$ semanas \\
\hline Carlucci Jr ${ }^{19}$ & 2007 & Alteração hormonal & $32,8 \%$ & $<8$ semanas \\
\hline Araújo Filho & 2007 & Alteração hormonal & $22,7 \%$ & 4-12 semanas \\
\hline
\end{tabular}


É importante ressaltar que é necessário um acompanhamento hormonal e clínico prolongado após a lobectomia da tireóide, pois, alterações hormonais e/ou recidiva da doença no lobo contralateral podem ocorrer tardiamente ${ }^{6,7}$.

Há diversos estudos que buscam determinar fatores preditores de hipotireoidismo após as tireoidectomias; muitos deles com resultados conflitantes. Alguns associam a presença de anticorpos antitireoideanos, sobretudo o anti-microssomal, a um risco maior de hipotireoidismo pós-operatório $0^{6,10,11}$. Outros autores, contudo, não estabeleceram esta relação ${ }^{8}$. Alguns pacientes apresentam níveis séricos de TSH pré-operatórios elevados, o que parece predizer um risco maior de desenvolverem hipotireoidismo no período pós-operatório, possivelmente devido a uma reserva funcional menor da tireóide ${ }^{4,9}$.

Alguns autores relatam uma associação bastante significativa entre a presença de infiltração linfocitária da tireóide e o hipotireidismo desenvolvido após as lobectomias ${ }^{6,8,12}$. Piper encontrou relação entre infiltrado linfocitário e hipotireoidismo pós lobectomia, porém essa relação era idade dependente ${ }^{13}$. Outros autores, contudo, não encontraram essa relação $0^{3,4,11,14-16}$.

O volume de glândula remanescente tem sido classicamente estudado como indicador de hipotireoidismo pósoperatório, em pacientes submetidos à tireoidectomia por bócio tóxico ${ }^{3,17-19}$. Alguns autores, no entanto, também consideram esta variável importante no tratamento de bócios simples, com maior ocorrência de hipotireoidismo em remanescentes menores que 8 gramas $^{8,15,16}$ ou $4 \mathrm{ml}^{19}$.

O tipo de doença da tireóide envolvida também tem sido associado à evolução do lobo remanescente ${ }^{20}$. Noel ob- servou aumento de níveis de TSH pós-operatório em $74 \%$ de pacientes submetidos à tireoidectomia subtotal por bócio multinodular simples e em $23 \%$ daqueles por bócio uninodular simples ${ }^{21}$.

Acreditamos, assim como diversos autores, que os pacientes com elevação nos níveis do TSH pós-operatório devam ser submetidos à terapia de reposição com hormônios tireoidianos, a fim de cessar o estímulo à glândula ${ }^{4,7,21,22}$.

É fundamental ressaltar que os níveis de hipotireoidismo após as lobectomias encontrados não inviabilizam o procedimento em relação à tireoidectomia total. Quando comparados os dois procedimentos, a lobectomia apresenta não apenas menores taxas de hipotireoidismo, mas também menor risco de lesão das paratireóides e do nervo laríngeo recorrente. A secreção hormonal do tecido tireoideano remanescente, mesmo que insuficiente, confere um comportamento mais fisiológico do que a reposição exógena pura. Além disso, caso haja recidiva da doença no lobo remanescente, o tratamento cirúrgico pode não ser mandatório.

Por fim, a ocorrência de elevação precoce dos níveis de TSH freqüente nos pacientes submetidos às lobectomias, chama atenção para a importância da avaliação hormonal no período pós-operatório. Há indícios de não haver relação entre sexo, idade e presença de tireoidite com elevação precoce do TSH após as lobectomias. Uma vez que as alterações hormonais podem ocorrer apenas tardiamente, recomendamos um seguimento a longo prazo, que é importante também, para diagnóstico o de eventuais alterações no tecido glandular remanescente.

\begin{abstract}
Background: To determine the frequency of serum elevations of thyrotropin in patients submitted to lobectomy within a period of up to 12 weeks after surgery, in the search for factors associated with its occurrence. Methods: Eighty-eight patients submitted to thyroid lobectomy from September 2002 to September 2004 in the Department of Head and Neck Surgery University of São Paulo Medical School were retrospectively analyzed. Thyroid hormone determinations were performed from 4 weeks on after the surgery. Cases of patients with altered preoperative hormone determination, need for total thyroidectomy, and loss of postoperative follow-up were excluded. Data regarding age and gender of patients, presence of thyroiditis on histopathologic analysis of the thyroid and time of emergence of hypothyroidism were studied. Statistical analysis was performed using Pearson's chi-square test. Results: Of the 88 patients, 71 (80.7\%) were women. The mean age was of 41.7 years. Elevation of thyrotropin was observed in 20 (22.73\%) of the 88 studied patients. No statistically significant difference was observed regarding its incidence in relation to gender, age or the presence of thyroiditis. Conclusion: Elevation of thyrotropin is frequent after thyroid lobectomy and it may occur early after surgery. In this study, no factor that could predict its occurrence in the short term were found.
\end{abstract}

Key words: Thyrotropin; Hypothyroidism; Thyroidectomy; Thyroiditis.

\section{REFERÊNCIAS}

1. Benediktsson R, Toft AD. Management of the unexpected result: compensated hypothyroidism. Postgrad Med J. 1998;74 (878):729-32.

2. Hedman I, Jansson S, Lindberg S. Need for thyroxine in patients lobectomised for benign thyroid disease as assessed by followup on average fifteen years after surgery. Acta Chir Scand. 1986;152:481-6.

3. Sugino K, Mimura T, Toshima K, Iwabuchi H, Kitamura Y, Kawano M, Ozaki O, Ito K. Follow-up evaluation of patients with Graves' disease treated by subtotal thyroidectomy and risk factor analysis for post-operative thyroid dysfunction. J Endocrinol Invest. 1993;16(3):195-9.

4. McHenry CR, Slusarczyk SJ. Hypothyroidisim following hemithyroidectomy: incidence, risk factors, and management. Surgery. 2000;128(6):994-8.

5. Vagenakis AG, Braverman LE, Azizi F, Portinay GI, Ingbar SH. Recovery of pituitary thyrotropic function after withdrawal of prolonged thyroid-suppression therapy. N Engl J Med. 1975;293(14):681-4.

6. Buchanan MA, Lee D. Thyroid auto-antibodies, lymphocytic infiltration and the development of post-operative hypothyroidism following hemithyroidectomy for non-toxic nodular goitre. J R Coll Surg Edinb. 2001;46(2):86-90. 
7. Cheung P, Boey J, Wong J. Thyroid function after hemithyroidectomy for benign nodules. World J Surg. 1986;10(4):718-23.

8. Bang U, Blichert-Toft M, Petersen PH, Nielsen BB, Hage E, Diederichsen $\mathrm{H}$. Thyroid function after resection for non-toxic goitre with special reference to thyroid lymphocytic aggregation and circulating thyroid autoantibodies. Acta Endocrinol. 1985;109(2):214-9.

9. Miller FR, Paulson D, Prihoda TJ, Otto RA. Risk factors for the development of hypothyroidism after hemithyroidectomy. Arch Otolaryngol Head Neck Surg. 2006;132(1): 36-8.

10. Chou FF, Wang PW, Huang SC. Results of subtotal thyroidectomy for Graves' disease. Thyroid. 1999;9(3):253-7

11. Jortso E, Lennquist S, Lundstrom B, Norrby K, Smeds S. The influence of remnant size, antithyroid antibodies, thyroid morphology, and lymphocyte infiltration on thyroid function after subtotal resection for hyperthyroidism. World J Surg. 1987;11(3):365-71.

12. Berglund J, Bondeson L, Christensen SB, Tibblin S. The influence of different degrees of chronic lymphocytic thyroiditis on thyroid function after surgery for benign, non-toxic goitre. Eur J Surg. 1991;157(4):257-60.

13. Piper HG, Bugis SP, Wilkins GE, Walker BAM, Wiseman S, Baliski CR. Detecting and defining hypothyroidism after hemithyroidectomy. Am J Surg. 2005;189(5):587-91; discussion 591.

14. Griffiths NJ, Murley RS, Gulin R, Simpson RD, Woods TF, Burnett D. Thyroid function following partial thyroidectomy. Br J Surg. 1974;61(8):626-32.

15. Berglund J, Aspelin P, Bondeson AG, Bondeson L, Christensen $\mathrm{SB}$, Ekberg O, Nilsson P. Rapid increase in volume of the remnant after hemithyroidectomy does not correlate with serum concentration of thyroid stimulating hormone. Eur J Surg. 1998;164(4):257-62.
16. Cusick EL, Krukowski ZH, Matheson NA. Outcome of surgery for Graves' disease re-examined. Br J Surg. 1987;74(9):780-3.

17. Taylor GW, Painter NS. Size of the thyroid remnant in partial thyroidectomy for toxic goitre. Lancet. 1962;1:287-9.

18. Allannic H, Guilhem I, Maugendre D. Is thyroid hormone useful in the prevention of nodular recurrence after hemithyroidectomy? Ann Endocrinol. 1993;54(4):286-90.

19. Carlucci Jr D. Função tireóidea após lobectomia total por bócio não tóxico. [Dissertação]. São Paulo: Universidade de São Paulo; 2007.

20. Niepomniszcze H, Garcia A, Faure E, Castellanos A, del Carmen Zalabar M, Bur G, Elsner B. Long-term follow-up of contralateral lobe in patients hemithyroidectomized for solitary follicular adenoma. Clin Endocrinol. 2001;55(4):509-13.

21. Noel P, Beckers C. TSH increase after subtotal thyroidectomy for nontoxic goiter. Ann Endocrinol. 1978; 39(2):163-4.

22. Cooper DS. Clinical practice. subclinical hypothyroidism. N Engl J Med. 2001;345(4):260-5.

Como citar este artigo:

Araújo Filho VJF, Brandão LG, Carlucci D, Moysés RA, Brescia MEG, Ferraz AR.

Elevação de hormônio tireoestimulante (TSH) em pacientes submetidos à lobectomia de tireóide: incidência e fatores associados. Rev Col Bras Cir. [periódico na Internet] 2007; 34(2). Disponível em URL: http://www.scielo.br/rcbc

Endereço para Correspondência:

Prof.Dr. Vergilius José Furtado de Araújo Filho

Av. Angélica, 1814 - Conj.601 - Higienópolis

01228-200 - São Paulo - SP

Telefone: + 55-11-3661-9137

e-mail: vergilius@globo.com 